\title{
'OHANA phase II: a prototype demonstrator of fiber linked interferometry between very large telescopes
}

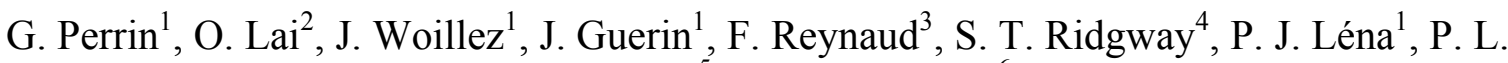 \\ Wizinowich $^{5}$, A. T. Tokunaga ${ }^{6}$, \\ J. Nishikawa ${ }^{7}$, F. J. Rigaut ${ }^{8}$, A. J. Adamson ${ }^{9}$, O. Guyon $^{6}$ \\ ${ }^{1}$ Observatoire de Paris, France \\ ${ }^{2}$ Canada-France-Hawaii Telescope Corporation, USA \\ ${ }^{3}$ IRCOM, Université de Limoges, France \\ ${ }^{4}$ National Optical Astronomy Observatory, USA \\ ${ }^{5}$ W.M. Keck Observatory, USA \\ ${ }^{6}$ Institute for Astronomy, University of Hawai'i, USA \\ ${ }^{7}$ National Astronomical Observatory of Japan, Japan \\ ${ }^{8}$ Gemini Observatory, USA \\ ${ }^{9}$ United Kingdom Infrared Telescope, GB
}

\begin{abstract}
The 'OHANA (Optical Hawaiian Array for Nanoradian Astronomy, means « family » in Hawaiian) aims at making a large and sensitive optical/IR array with the Mauna Kea 3 to 10 meter telescopes. Telescopes will be linked with single-mode fibers to carry the coherence of the beams from the output of the telescopes adaptive optics systems to the beam combination units. The project has been divided into three phases. The first phase is dedicated to the injection of light into single-mode fibers and to the building of the injection module. The third phase is the realization of the complete array and its use by a wide community of astronomers. In the second phase, a prototype 'OHANA will be built and the "shortest » baselines will be explored. The baselines will be located in the SouthEast and West parts of the observatory. An extra baseline will possibly link the two groups of telescopes if infrastructure comply with it. This phase II 'OHANA will already be the longest and most sensitive optical/IR interferometer built. Scientific targets will span young stellar objects, extragalactic sources and other types of astronomical topics which require both high angular resolution and sensitivity. This paper reviews the main characteristics of the phase II interferometer.
\end{abstract}

Keywords: Interferometry, OHANA, Singlemode fiber, Adaptive Optics

\section{INTRODUCTION}

The 'OHANA project consists in linking the large telescopes of the Mauna Kea summit with single-mode fiber optics to realize a large near-infrared interferometer. The idea was first published by Mariotti et al. (1996) ${ }^{1}$. The project started in 2000 and has been described in its current version in Perrin et al. (2000) ${ }^{2}$. A similar idea was proposed to link the Subaru and the Gemini telescopes by Nishikawa et al. (1998) ${ }^{3}$. 'OHANA is a quite ambitious and new type of interferometer. Several phases are therefore necessary before a full combination of the Mauna Kea telescopes can be achieved. This is why the project has been divided into three phases. A first phase to build and optimize the instruments required to inject light into single-mode fibers at the focus of the adaptive optics systems. This phase is in progress and first tests at CFHT are described in Woillez et al. (2002) ${ }^{4}$. In the second phase described in this paper, the "shortest" baselines will be linked to demonstrate the concept of 'OHANA in the J, H and $\mathrm{K}$ bands. Once it is believed that 'OHANA can be operated to produce very valuable astrophysical results, the full array may be built and operated as described in Lai et al. (2002) ${ }^{5}$. We present the main features of the phase II 
instrument in the following paragraphs. It is a prototype instrument and not a general user facility as VLTI and the Keck interferometer.

\section{BASELINES}

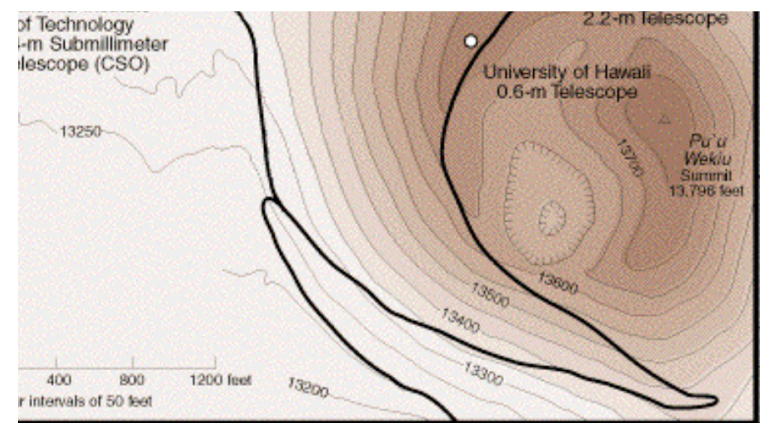

Figure 1: Map of the Mauna Kea summit. The red and blue lines represent the 'OHANA phase II baselines. The green area is the future phase III full 'OHANA array. The dashed purple line connects CFHT to IRTF a possible although very difficult baseline for phase II. In the bottom left corner VLTI is represented at the same scale.

Figure 1 shows a map of the Mauna Kea observatory. The solid and dashed lines are the baselines conceivable during phase II. Except for the Keck telescopes, none of the other telescopes have been designed to be used interferometrically. As a consequence they are not equipped with facilities to equalize the optical paths which therefore has to be done. Because of the telescopes lay-out on the mountain, it is possible to distinguish two groups of telescopes : the telescopes roughly aligned in a North-South direction as UKIRT, Gemini and CFH, and the telescopes aligned in an East-West direction as Subaru, Keck I\&II and IRTF. It is very fortunate that the Keck telescopes are situated in the middle of the East-West complex. As a matter of fact, it is very difficult to find and track fringes on an East-West baseline and this requires very good and efficient delay lines. The Keck interferometer delay lines are therefore ideally suited for these baselines. On the other side, a dedicated delay-line will have to be fabricated for the quasi North-South baselines. This delay line will first be located in the CFHT Coudé room for the coupling of Gemini and CFHT, the first baseline of 'OHANA as it is one of the shortest baselines and has a favourable orientation. The delay line is described in Section 4. The IRTF-CFHT baseline is represented by a dashed line as it is not sure yet that either enough lengths of fibers or suitable delay lines will be available at this stage of the project. These baselines altogether provide a very good coverage in azimuth in spatial frequency space. The green area represents the baselines achievable in phase III when the longest baseline (Gemini-Subaru, 800m) will provide the highest spatial resolution.

The table below lists the baseline lengths and the spatial resolutions in the $\mathrm{J}, \mathrm{H}$ and $\mathrm{K}$ bands. The colours are matched to that of the groups of baselines on the figure above. All baselines are longer than what both the Keck interferometer and the VLTI can provide at equivalent sensitivity. 'OHANA phase II will therefore be a good 
complement to these two projects and it will extend their resolving power by a factor of 3 . Due to the supersynthesis effect, objects of a few 0.1 mas will be resolved by the interferometer.

\begin{tabular}{|l|c|c|c|c|}
\hline \multirow{2}{*}{ Baseline } & \multirow{2}{*}{ Length $(\mathrm{m})$} & \multicolumn{3}{|c|}{ Resolution (mas) } \\
\cline { 3 - 5 } & & $\mathrm{J}$ & $\mathrm{H}$ & $\mathrm{K}$ \\
\hline Keck I-Subaru & $\mathbf{1 4 7}$ & $\mathbf{1 . 7 5}$ & $\mathbf{2 . 3 2}$ & $\mathbf{3 . 0 9}$ \\
\hline Keck II-Subaru & $\mathbf{2 2 2}$ & $\mathbf{1 . 1 6}$ & $\mathbf{1 . 5 3}$ & $\mathbf{2 . 0 5}$ \\
\hline IRTF-Keck II & 237 & $\mathbf{1 . 0 9}$ & $\mathbf{1 . 4 3}$ & $\mathbf{1 . 9 1}$ \\
\hline IRTF-Keck I & $\mathbf{2 8 7}$ & $\mathbf{0 . 9 0}$ & $\mathbf{1 . 1 9}$ & $\mathbf{1 . 5 8}$ \\
\hline IRTF-Subaru & $\mathbf{4 3 0}$ & $\mathbf{0 . 6 0}$ & $\mathbf{0 . 7 9}$ & $\mathbf{1 . 0 6}$ \\
\hline CFHT-IRTF & 344 & $\mathbf{0 . 7 5}$ & 0.99 & 1.32 \\
\hline CFHT-Gemini & 162 & 1.59 & 2.10 & 2.80 \\
\hline UKIRT-Gemini & 202 & 1.28 & 1.68 & 2.25 \\
\hline CFHT-UKIRT & 347 & $\mathbf{0 . 7 4}$ & $\mathbf{0 . 9 8}$ & 1.31 \\
\hline
\end{tabular}

\section{FIBERS}

'OHANA will use two kinds of single-mode fibers: silicate and fluoride glass fibers. The silicate fibers will be used for the $\mathrm{J}$ and $\mathrm{H}$ bands whereas the fluoride glass fibers will give access to the $\mathrm{K}$ band. The losses of these fibers are in the range $0.1-0.2 \mathrm{~dB} / \mathrm{km}$ for the silicate fibers in $\mathrm{J}$ and $\mathrm{H}$ and $1-2 \mathrm{~dB} / \mathrm{km}$ for the fluoride glass fibers in the $\mathrm{K}$ band, $3 \mathrm{~dB}$ meaning a loss of $50 \%$ of the photons. Connectors between fibers now have losses of $0.1 \mathrm{~dB}$. The maximum length of fibers to be used for phase II is about $500 \mathrm{~m}$. Gemini and Keck could be used to host the beam combiner and delay line hence reducing the maximum length of fiber to half a baseline for the UKIRT-CFHT and Subaru-IRTF connections. The maximum loss caused by propagation in the fibers is therefore of $1 \mathrm{~dB}$ for baselines up to $450 \mathrm{~m}$ and a conservative value of the transmission is of $50 \%$ which is very competitive compared to classical optical trains to guide the beams. Besides, transmission and coherence losses created by diffraction effects disappear in fibered links. Yet, bulk optics will have to be used for the delay lines as long delays cannot be produced by stretching fibers without creating differential dispersion. Extra losses will therefore occur when getting out and back in the fibers.

Fibers are known to be dispersive media and this point has to be addressed cautiously to build the interferometer. It is basically impossible today to cancel intrinsic dispersion in fibers as this requires to master the waveguide design to within a terrific precision (on the order of a few nanometers on the core size for example). The technique used consists in adjusting the fiber lengths and choosing the fiber cables so that intrinsic dispersions are matched in the two lengths of fibers in the interferometer. This has proved to be efficient on fibers a few meters long where the dominant effect (second order) can be cancelled by adjusting the respective lengths of the fibers. For longer fiber lengths, the second order of dispersion can still be adjusted with this technique but the third order is no more negligeable. The third order is basically generated by the variation of the core diameter along fiber cables. It can be cancelled by breaking a long fiber into shorter fibers a few tens or a hundred meters long and by choosing the best combination.

Another matter of concern to address are polarization effects. We will use polarization maintaining fibers in the $\mathbf{J}$ and $\mathrm{H}$ bands. One of the polarization will be filtered to detect fringes as the two linear polarizations do not travel at the same velocity hence the interferograms are not synchronized and need to be separated. Standard fibers will have to be used in the $\mathrm{K}$ band as no such components are available for long distance links. Yet, fluoride glass fibers have a very small birefringence so that polarization effects should amount to a differential rotation of the polarization axes which can be compensated by twisting the fibers.

The fibers used for 'OHANA will have to be protected with flexible cables. They will be plugged-in at the foci of the injection modules and will run to the telescopes enclosures housing the delay lines and the beam combining systems. For the very first baselines of phase II, fibers can be put on the ground. In later stages of phase II, they will have to be pulled from existing ducts used for phone and network cables between telescopes. The same sets of fibers 
will be used for all the baselines. For the shortest baselines, $300 \mathrm{~m}$ of fibers are required for a focus-to-beam combiner connection. 2x500 m of fibers per band will be enough to realize all the baselines (but observations cannot be done simultaneous with different baselines).

\section{DELAY LINES}

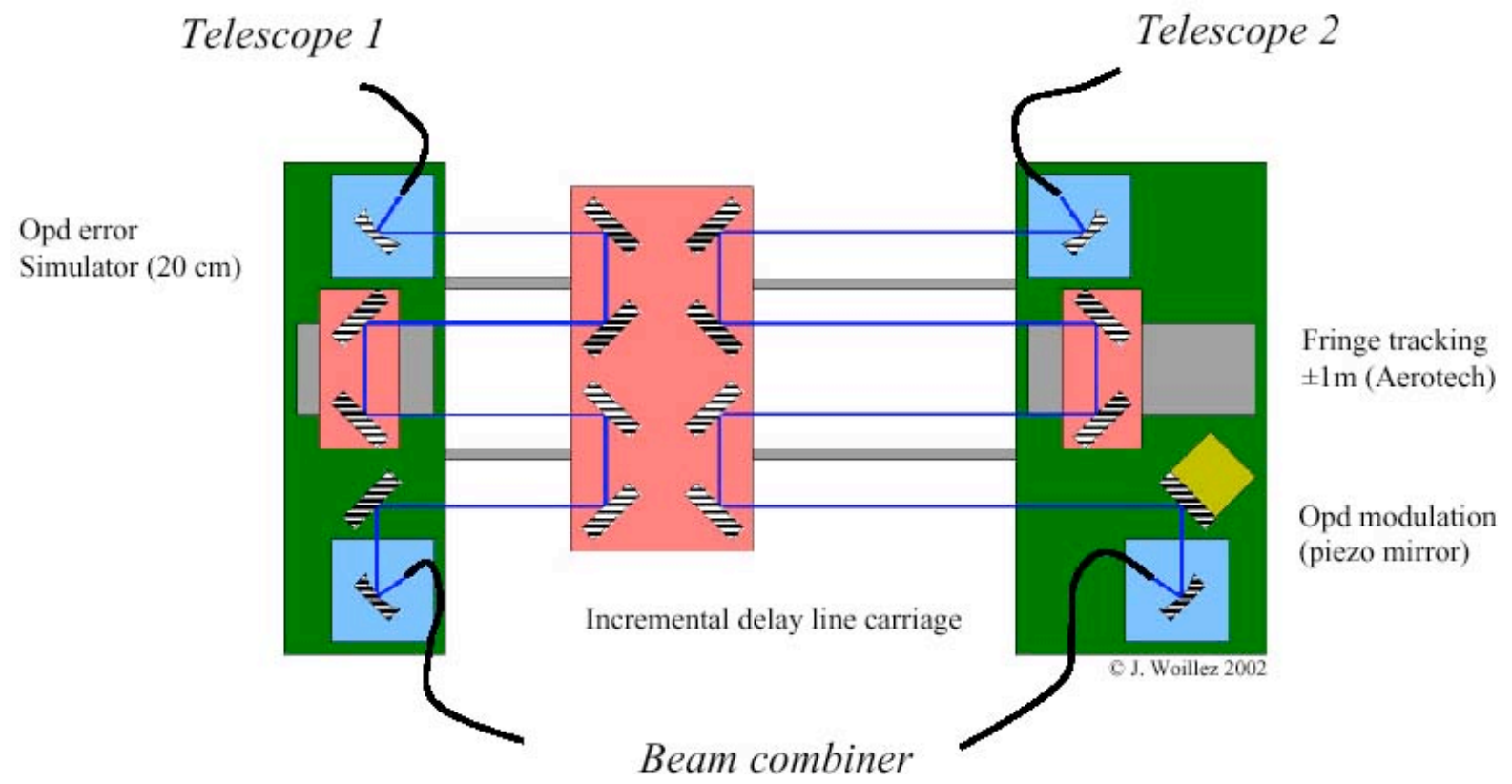

Figure 2: delay line concept for the phase II of 'OHANA. The two J, H and K single-mode fiber bundles coming from Telescope 1 and 2 are connected at the focus of an off-axis parabola. The collimated beams are then reflected by three di-hedrals and focused again in single-mode fibers connected to the beamcombiner. The delay line is a three stage system. The central carriage is moved between observations to compensate for the gross of the delay. It pushes in one arm and pulls in the other. The fringe tracking delay line follows the natural fringe drift during an observation and the flat mirror actuated by a piezo scans through fringe packets. The opd error simulator is used to simulate a fringe drift during test phases. The total stroke of the delay line is 50 meter.

Delay lines are a critical issue at Mauna Kea. No extra builiding can be built for 'OHANA forbiding the realization of new Keck-like delay lines. Fortunately, Keck is part of the array and provides a solution for the baselines including telescopes nearby Keck and the Keck telescopes (red baselines on Figure 1). For the western part of the array, baselines are quasi North-South oriented hence the fringe speed remains under $20 \mathrm{~mm} / \mathrm{s}$ even on the longest UKIRT-CFHT baseline as can be seen on Figure 3. A simple system can be designed to provide enough delay and track the fringes for the UKIRT-Gemini-CFHT baselines. It is a three stage system represented on Figure 2.The beams are output from the fibers with off-axis parabolas and relayed by flat and dihedral mirrors to off-axis parabolas where they are refocused in fibers. A gross delay is produced by two dihedrals mounted on a translation stage common to the two beams (two dihedrals per beam) allowing to produce $+/$ - the maximum stroke of delay $(+/-$ $50 \mathrm{~m})$. With the four-pass system, the translation of the dihedrals will produce a delay four times larger than the extension of the carriage thus not requiring more than 13 meters of space. This four dihedral carriage will be still during observations. An extra offset in delay can be produced if necessary by putting one of the input or output parabolas within a fixed distance of their nominal position to shift the zero optical path difference black strip in Figure 3. This will allow to extend the sky coverage for the western baselines.

Fringes will be tracked with a fast dihedral mounted on a commercial 1m-stroke translation stage in one of the arms of the interferometer. In the same arm, a flat mirror is mounted on a piezo-stage to produce the modulation of the 
fringe packet. In the other arm, the third dihedral sits on a short stroke translation stage used to simulate baseline errors to exercize fringe tracking.

This system is designed to be quickly reconfigurable with fast alignment procedures when translating the incremental delay dihedrals $(30 \mathrm{~s}$ to $1 \mathrm{~min})$. It is primarily designed for the first baseline Gemini-CFHT and it will be installed in the CFHT Coudé room. As shown on the figure below, this system can be used for the CFHT-UKIRT baseline. Other designs are proposed for longer baselines by Ridgway et al. (2002) 6 in this coneference.

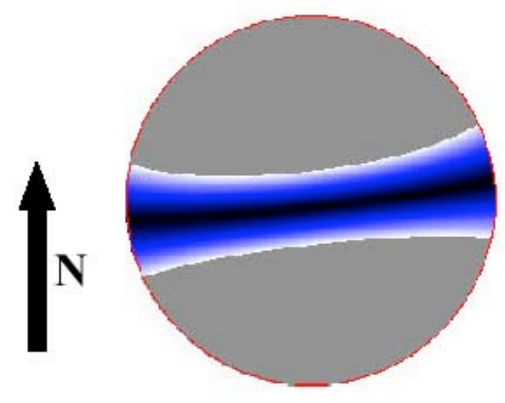

Optical path difference $(\max =50 \mathrm{~m})$

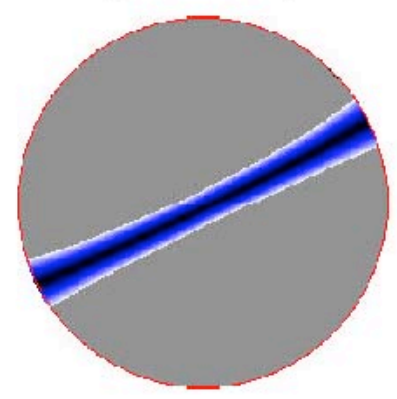

GEMINI-CFH

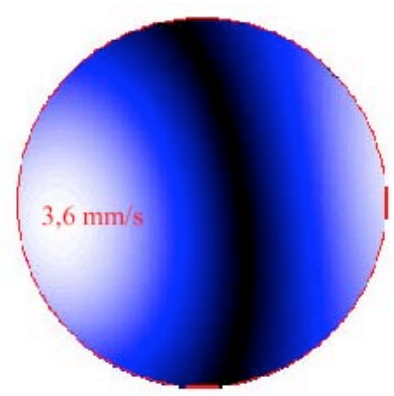

Fringe speed $(\max =20 \mathrm{~mm} / \mathrm{s})$

UKIRT-CFH

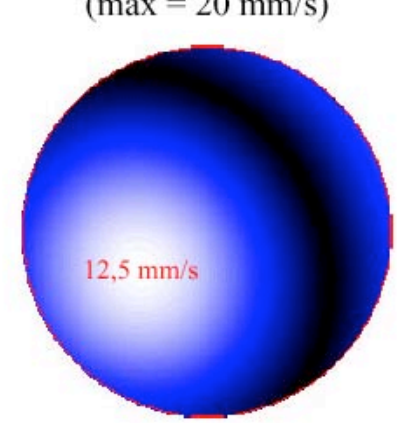

Figure 3: optical path difference and fringe speed projected on the sky for the GEMINI-CFHT and UKIRT-CFHT baselines. The colour-coded scales go from black (0 speed or optical difference) to white. The graphs on the left represent the regions of the sky that can be accessed with a maximum differential delay of $50 \mathrm{~m}$. North points to the top. The non-grey areas represent the parts of the sky that can be accessed with the simple delay line presented in this paper. The graphs on the right are the corresponding fringe speeds. The maximum speed is smaller on both cases than the maximum tracking speed of the continuous carriage of $20 \mathrm{~mm} / \mathrm{s}$. Fringes can be tracked for a given a source for more than 30 minutes in average.

\section{SENSITIVITY AND ASTRONOMICAL PROGRAM}

The sensitivity estimate depends on the transmission of the whole system and on the stability of the injection in the fibers. Allowing these two parameters to vary within realistic ranges and assuming $1 \mathrm{e}^{-}$read out noise for the detector a limiting magnitude of $K=13 \pm 1$ can be derived with the 8 meter telescopes ${ }^{2}$. This estimate will be refined during phase I and at the beginning of phase II during integration of the system. First tests at CFH are compatible with this estimate $^{4}$. If fringe tracking on an off-axis source is possible in phase II (not anticipated in the current state of the project) then this limiting magnitude can be extended by 5 magnitudes.

This sensitivity will allow to address programs very similar to that of Keck and VLTI: YSOs, AGNs, brown dwarves, ... and will provide both very complementary and unique data ${ }^{7,8,9}$. For example, the study of YSOs will benefit a lot from baselines reaching $400 \mathrm{~m}$ as they are dramatically needed to disentangle between disk models in 
the near infrared. More generally, merging these data with the Keck-VLTI data will create continuous data sets which will prove very useful to analyze the complex geometry of these objects.

\section{SCHEDULE}

'OHANA is now in its first phase. The first combination between CFHT and Gemini is anticipated for the second semester of 2003. The second baseline will couple two telescopes on the western side. The integration of the fibers will start in the beginning of 2003. The whole instrument should be tested in Meudon during the first semester of 2003. Other baselines will require injection modules to be built hence phase I to have been achieved for these telescopes. A reasonable schedule for these other telescopes is 2004 for first fringes.

\section{REFERENCES}

1. J.-M. Mariotti et al., "Interferometric connection of large ground-based telescopes", A\&AS 116, 381, 1996

2. G. Perrin et al., "A fibered large interferometer on top of Mauna Kea: 'OHANA, the Optical Hawaiian Array for Nano-radian Astronomy", SPIE 4006, 708, 2000

3. J. Nishikawa et al., "MIRA-II, MIRA-III, and MIRA-SG projects: the future plan of long-baseline optical/IR interferometers in Japan", SPIE 3350, 184, 1998

4. J. Woillez et al., "Injection into single-mode fibers for 'OHANA", SPIE 4838, this volume

5. O. Lai et al., "OHANA phase III: scientific operation of an 800-meter Mauna Kea interferometer", SPIE 4838, this volume

6. S.T. Ridgway, "Optical delay concepts for OHANA", SPIE 4838, this volume

7. O. Lai et al., "OHANA: representative science objectives", SPIE 4838, this volume

8. J. Woillez et al., "Extragalactic astronomy with the OHANA array", SPIE 4838, this volume

9. F. Ménard et al., " Protoplanetary disks studied with OHANA", SPIE 4838, this volume 